Les dictionnaires bilingues des maîtres ou professeurs auteurs de manuels pédagogiques

\title{
Rafael Reyes et ses dictionnaires français-espagnol et espagnol-français (1926-1928)
}

\section{Manuel Bruña Cuevas}

\section{(2) OpenEdition \\ Journals}

Édition électronique

URL : https://journals.openedition.org/dhfles/3994

DOI : $10.4000 /$ dhfles.3994

ISSN : 2221-4038

Éditeur

Société Internationale pour l'Histoire du Français Langue Étrangère ou Seconde

Édition imprimée

Date de publication : 1 juin 2016

Pagination : 189-206

ISSN : 0992-7654

Référence électronique

Manuel Bruña Cuevas, « Rafael Reyes et ses dictionnaires français-espagnol et espagnol-français

(1926-1928) », Documents pour l'histoire du français langue étrangère ou seconde [En ligne], 56 | 2016, mis en ligne le 03 décembre 2017, consulté le 31 mars 2023. URL : http://journals.openedition.org/ dhfles/3994 ; DOI : https://doi.org/10.4000/dhfles.3994

Ce document a été généré automatiquement le 31 mars 2023.

Tous droits réservés 


\title{
Rafael Reyes et ses dictionnaires français-espagnol et espagnol- français (1926-1928)
}

\author{
Manuel Bruña Cuevas
}

1 Cette étude porte sur la vie et l'œuvre de Rafael Reyes. Étant donné que la trajectoire professionnelle de cet auteur n'a pas été abordée en profondeur jusqu'à présent et qu'elle a eu une incidence directe sur sa production didactique, nous tracerons d'abord une biographie de Reyes, ce qui nous permettra de repérer aussi bien les traits de son activité en commun avec d'autres professeurs du secondaire que ceux qui lui sont propres. Nous y situerons la publication de son dictionnaire, auquel nous consacrerons la seconde partie de ce travail.

\section{Biobibliographie de l'auteur}

2 Rafael Reyes Rodríguez est né le 7 avril 1879 à Cartaya, une localité andalouse de la province de Huelva. Il est mort en $1947^{1}$.

\subsection{Sa première étape}

3 II commence sa vie professionnelle à l'âge de vingt ans; dès 1899, il accède par concours au corps des télégraphistes ${ }^{2}$, ce qui revient à dire qu'il a été obligé d'acquérir un certain niveau en langues étrangères. C'est cela qui peut expliquer qu'il ait fini par consacrer sa vie professionnelle à l'enseignement de l'anglais et du français, se décidant bientôt, comme nous le verrons, pour cette dernière langue. En effet, après en avoir donné des cours dans des établissements privés ${ }^{3}$ et avoir collaboré avec quelques journaux et revues ${ }^{4}$, il devient, également par voie de concours national, enseignant du secteur public, d'où sa nomination le 17 mars 1906 comme professeur de langue française de l'école technique de Saint-Jacques-de-Compostelle ${ }^{5}$. C'est justement cette année-là qu'il publie ses deux premiers ouvrages didactiques pour le secondaire : une 
grammaire française (Gramática sucinta, deux cours) ${ }^{6}$ et une anthologie de textes littéraires (Trozos de literatura francesa). d'anglais à l'école technique de la même ville, ce qui pourrait expliquer que, sans délaisser ses publications pour l'apprentissage du français ${ }^{8}$, il ait décidé de publier en 1910 une grammaire de l'anglais (Gramática sucinta de lengua inglesa) qui fera figure d'exception dans sa production, étant donné que, postérieurement, toutes ses publications seront consacrées au français ; cette grammaire ne sera d'ailleurs jamais rééditée, ce qui contraste avec les nombreuses réimpressions de ses autres ouvrages. Cette publication indique, toutefois, que, pour lors, il ne voyait pas encore son avenir comme essentiellement lié au français.

6 En effet, ayant obtenu à Madrid une licence de Lettres, mention Histoire, il sollicite le 23 février 1911 à la Junta para Ampliación de Estudios ${ }^{9}$ une bourse pour faire un stage à Londres, où il pourrait consulter les archives du Foreign Office dans le but de compléter un travail de recherche sur les relations diplomatiques de l'Espagne pendant la dernière régence $(1885-1902)^{10}$. Bien que l'auteur prévoie dans sa demande la publication de son travail par le Centro de Estudios Históricos, celle-ci n'a jamais eu lieu, certainement du fait que la bourse à laquelle il aspirait ne lui a pas été accordée ${ }^{11 .}$ l'enseignement ne lui a plus suffi. D'une part, étant aussi licencié en Droit ${ }^{12}$, dès décembre 1918 il s'occupera comme avocat, pendant plusieurs années, d'une affaire retentissante sur un meurtre, ce qui, ajouté à sa charge professorale et à l'édition de ses œuvres, le conduira, selon ses propres mots, à un état de "surmenage " (La Acción, 24/11/1920, p. 6). D'autre part, il fera sa seconde demande de congé sans solde, qu'il obtiendra le 7 avril $1919^{13}$. Fort probablement, il s'apercevait déjà des possibilités économiques que lui offrait l'édition d'ouvrages destinés à l'enseignement du français dans le secondaire, car il a profité de ce congé pour préparer la publication d'une nouvelle anthologie (El traductor francés, 1922), qu'il présente comme susceptible de servir de livre de texte pour la pratique de la traduction.

\subsection{Son étape à Séville}

Lorsqu'il sollicite au ministère sa réintégration dans l'enseignement secondaire, il vise en réalité l'obtention d'un poste dans un des lycées espagnols les plus réputés. Sachant que le professeur de français de ce lycée devait passer à la retraite le $1^{\text {er }}$ juin 1921, il fait sa demande de fin de congé en mai pour s'assurer que le prochain poste vacant serait celui de Séville, qu'il sollicite, en effet, le 4 juin. Malheureusement pour lui, le ministère a autrement interprété la loi sur la distribution des postes vacants. Reyes, ayant su que les autorités ministérielles pensaient l'assigner au lycée de Lugo, a adressé le 9 juin un troisième écrit au ministère où il revendiquait son droit au poste de Séville ou, autrement, la continuation de sa mise en disponibilité (Gaceta de Madrid, 2/8/1921). Comme le ministère a nommé Joaquín López Barrera ${ }^{14}$ à Séville, Reyes est resté en congé volontaire, quoique non sans faire appel aux tribunaux contre l'arrêté 
ministériel. La justice lui a finalement donné raison, ce qui lui a valu d'être nommé professeur de français au lycée de Séville le 18 avril 1922 (Gaceta de Madrid du 24) ${ }^{15}$.

9 Son étape à Séville va marquer l'édition de ses ouvrages. C'est dans cette ville, et non plus à Madrid, que seront imprimées plusieurs rééditions ${ }^{16}$ des deux niveaux de sa grammaire française ${ }^{17}$, de son anthologie de textes littéraires ( $\left.{ }^{7} 1926\right)$ et de son Traductor $\left({ }^{2} 1923,{ }^{3} 1924\right)$. L'auteur cherchait sans doute par-là à mieux surveiller l'impression de ses œuvres; cependant, il recommencera bientôt à les faire imprimer à Madrid, où il disposait aussi d'une résidence (71, rue Meléndez Valdés à cette époque) ${ }^{18}$. C'est à Madrid, en fait, que seront publiés ses nouveaux titres : un nouveau manuel du français (Curso práctico de francés, 1923) et un dictionnaire français-espagnol (1926) et espagnolfrançais (1928).

10 Le 7 septembre 1932, Reyes adresse une nouvelle demande à la Junta para Ampliación de Estudios: il souhaite en avoir la tutelle officielle pour faire un stage à l'Institut hispanique de Paris ${ }^{19}$. Cette fois-ci la Junta lui répond favorablement, ce qui lui permet, malgré quelques difficultés à lui trouver un remplaçant pour son poste au lycée de Séville, de passer huit mois sans solde - de novembre 1932 à juin 1933 - à Paris, Bordeaux et Toulouse afin d'améliorer - avait-il déclaré - la seconde partie de son dictionnaire, espagnol-français ${ }^{20}$. Par contre, la Junta n'accepte pas de renouveler son stage pour une période de la même durée, comptée à partir de novembre $1933^{21}$. Ces demandes montrent bien que Reyes ne dépendait pas de son traitement comme enseignant pour pouvoir vivre; il est probable que ses diverses publications lui procuraient les ressources suffisantes pour se permettre de s'en passer.

11 Pour des raisons qui nous échappent, une fois revenu à son poste de professeur à Séville il sollicite et obtient deux mutations successives : le 26 juillet 1933 (Gaceta de Madrid du 3 août), il est affecté au lycée de Gijón et, le 26 septembre de cette même année (Gaceta de Madrid du 5 octobre), à celui d'Elche. Mais, en fait, il n'y restera pas longtemps. En février 1934 il obtient un congé de maladie d'un mois, auquel il ajoutera une demande de congé sans solde (de mars à mai 1934). Après les mois d'été, il réussit à avoir une nouvelle mise en disponibilité dès le 29 octobre 1934 (Gaceta de Madrid du 9 novembre). Ce n'est que le 4 décembre 1935 que sa demande d'être réintégré à un lycée a été approuvée par le ministère, qui lui assigne le 25 mars 1936 (Gaceta de Madrid du 28) le poste de professeur de français du lycée de Ségovie, Reyes devant encore attendre jusqu'au $1^{\text {er }}$ juillet pour récupérer la totalité du salaire correspondant à sa catégorie professionnelle. Il a donc pratiquement quitté son activité comme enseignant pendant deux ans, ce qui indique une fois de plus qu'il n'en avait probablement pas besoin pour vivre, certainement du fait que les nouvelles rééditions de ceux de ses ouvrages déjà publiés et les nouveaux titres qu'il met sous presse pendant les premières années de la décennie 1930 lui rapportaient des revenus.

12 En effet, depuis 1930, Reyes s'occupe de mettre chaque année à la disposition des lycéens un nouveau tirage des ouvrages qu'il avait précédemment publiés : sa Gramática sucinta de lengua francesa ${ }^{22}$, son anthologie littéraire de morceaux choisis (Trozos de literatura francesa $\left.{ }^{23}\right)$, son Traductor $\left({ }^{1} 1922,{ }^{8} 1931,{ }^{9} 1932\right)$, son cours pratique (Curso práctico de francés, $\left.{ }^{1} 1923,{ }^{2} 1933,{ }^{3} 1934,{ }^{4} 1935\right)$ et son dictionnaire ${ }^{24}$. L'auteur en assume lui-même les coûts d'impression en les concertant avec diverses maisons madrilènes, ce qui, sans doute, devait lui prendre une grande partie de son temps et devait exiger sa présence à Madrid pour surveiller ses affaires, d'autant plus que, comme déjà dit, sur ces ouvrages il était indiqué que les commandes pouvaient être adressées à son 
domicile madrilène. On comprend donc qu'il ait eu besoin de solliciter au ministère plusieurs périodes de congé sans solde, rien que la vente de son dictionnaire, plus cher que le reste de ses œuvres et moins soumis à la concurrence d'ouvrages similaires, lui assurant une position économique aisée ${ }^{25}$. Mais à tout cela il faut ajouter que l'auteur, afin de garder sa part du marché des élèves du secondaire, a été obligé de remettre à jour sa production pour l'adapter au nouveau plan d'études de la République, instaurée en $1931^{26}$. De ce fait, il lance en 1936 une nouvelle grammaire française pour les lycéens (Primer curso del plan cíclico de francés), qui avait été précédée en 1933 d'une nouvelle anthologie (Lectures françaises graduées), deux fois réimprimée en 1934 et avec une quatrième édition en 1935) et des deux volumes d'une histoire de la littérature française par les textes ${ }^{27}$.

\subsection{Sa dernière étape}

13 Malheureusement, cette brillante trajectoire se verra altérée par la guerre civile espagnole de 1936-1939, qui divise le pays en deux zones, la républicaine, fidèle au régime établi, et celle des insurgés. Étant donné que le soulèvement militaire contre la République a eu lieu en période de vacances scolaires, il a probablement surpris notre auteur à son domicile madrilène ; or Madrid est resté en zone républicaine, alors que la ville où se trouvait le lycée auquel Reyes avait son poste, Ségovie, est passée dès le début sous le contrôle des forces insurrectionnelles. Il n'a donc pu (ni voulu ?) rejoindre son poste de travail ni dans les premiers jours de guerre ni, encore moins, à la rentrée de l'année scolaire 1936-1937. Devenu donc suspect pour les autorités académiques du régime antirépublicain du général Franco ${ }^{28}$, le 14 octobre 1937 celles-ci le déclarent suspendu de ses fonctions de professeur et lui interdisent également l'exercice de toute autre fonction dans un établissement scolaire ou culturel ${ }^{29}$. La guerre a donc été dure pour notre auteur. Il a encore édité ou réédité certains de ses ouvrages en 1936, mais, à une exception près, aucun d'entre eux ne reparaîtra avant 1941. En effet, seul son dictionnaire, pièce principale de sa collection d'ouvrages aussi bien du point de vue de son envergure que du point de vue économique, aura une nouvelle réédition pendant la période de la guerre d'Espagne. Après sa dixième réédition en 1936, notre auteur réussit à en faire imprimer la onzième à Paris en 1938. C'est la seule de ses 41 éditions à ne pas être sortie des presses madrilènes ${ }^{30}$ : dès ${ }^{12} 1940$, il reparaît à Madrid.

Une fois la guerre terminée en 1939 avec le triomphe des forces franquistes, Reyes fait appel contre l'arrêté qui l'avait condamné en 1937. Un nouvel arrêté daté du 11 juillet 1940 confirme la décision précédemment prise, mais l'atténue en décrétant sa mise à la retraite $^{31}$, finalement approuvée, avec la pension correspondante, le 8 février $1941^{32}$. Reyes était alors proche de ses 63 ans, ce qui nous porte à croire que ce dénouement, compte tenu des circonstances, n'a pas dû trop le déranger ; en tout cas, il lui a permis de se consacrer entièrement à l'édition de ses ouvrages, qu'il lui a fallu adapter encore à un nouveau plan d'études. Dès cette même année 1941 paraissent la $14^{\mathrm{e}}$ réédition de sa grammaire $\left({ }^{1} 1906\right)$, la $10^{e}$ de son anthologie littéraire $\left({ }^{1} 1906\right)$, la $5^{e}$ de ses Lectures françaises graduées ( $\left.{ }^{1} 1933\right)$ et la $2^{\mathrm{e}}$ de La literatura francesa ilustrada con textos ( $\left.{ }^{1} 1935-1936\right)$.

15 Jusqu'en 1947, date de son décès, Reyes ne cessera de rééditer ses livres. Ses héritiers, à leur tour, s'occuperont de continuer à les publier longtemps après la mort de l'auteur. La $16^{\mathrm{e}}$ édition de sa Gramática de lengua francesa $\left({ }^{1} 1906\right)$ paraît en 1945 , avec une nouvelle réédition finale en 1951 ; la $12^{\mathrm{e}}$ des Trozos littéraires ( $\left.{ }^{1} 1906\right)$ le fait en 1946 et 
arrive à la fin de son parcours avec celle de 1950 ; ses Lectures françaises ( $\left.{ }^{1} 1933\right)$, publiées pour la $7^{\mathrm{e}}$ fois en 1945 , arrivent jusqu'à leur $9^{\mathrm{e}}$ réédition en 1953 , et son histoire de la littérature française $\left({ }^{1} 1935\right)$, après sa réédition par l'auteur en 1946, reparait une dernière fois en 1952. Ces rééditions ne sont plus possibles à partir de 1953 du fait qu'un nouveau plan d'études est entré en vigueur cette année-là et que Reyes ne pouvait plus les remanier. Toutefois, le principal d'entre eux, son dictionnaire, n'était pas concerné par ce changement de plan, raison pour laquelle les héritiers de Reyes y ont consacré leurs efforts éditoriaux, le conduisant de la sorte jusqu'à sa $41^{\mathrm{e}}$ édition en $1979^{33}$.

\section{Le dictionnaire}

Comme nous l'avons indiqué, les deux parties du dictionnaire de Reyes n'ont pas été publiées ensemble dès le début. La première édition de la partie français-espagnol a été imprimée à Madrid en 1926; toutefois, elle portait déjà le titre de Diccionario francésespañol y español-francés, ce qui laissait prévoir la prochaine parution de sa deuxième partie, qui a eu lieu en $1928^{34}$. L'auteur a donc commencé son ouvrage par la partie la plus nécessaire pour les lycéens espagnols, qui devaient faire des lectures de textes en français et traduire de cette langue en espagnol ${ }^{35}$.

Dès la première édition, Reyes présente son ouvrage comme étant un dictionnaire manuel et bon marché («al alcance de todas las fortunas »). Ces deux caractéristiques sont en fait essentielles : ce sont elles qui assureront son succès grandissant parmi les élèves du secondaire, lesquels le garderont comme dictionnaire bilingue usuel dans leur âge adulte. Dès 1928, les deux tomes ont été vendus, jusqu'à l'édition de ${ }^{14} 1942$, soit en deux volumes, dont on pouvait n'en acheter qu'un, soit en un seul, dont le prix de vente (10 pesetas dans les années 1930) était légèrement moins élevé que si l'on acquérait les deux volumes séparément (5,5 pesetas chacun). Cette double possibilité et le fait que c'était l'auteur lui-même qui se chargeait de l'impression et de la distribution du dictionnaire lui permettaient de le commercialiser à ces prix, très compétitifs. Il a su, par ailleurs, profiter des circonstances historiques pour en faire la promotion. En 1929-1930 ont eu lieu en Espagne deux importantes expositions : l'Exposition ibéroaméricaine (Séville, du 9 mai 1929 au 21 juin 1930) et l'Exposition internationale de Barcelone (du 20 mai 1929 au 15 janvier 1930). L'édition de 1929 du dictionnaire se présente sur la page de titre de sa partie français-espagnol comme une édition spéciale pour ces deux événements; dès l'édition de 1930, on y lit la mention «Premier Prix : Médaille d'Or à l'Exposition Internationale de Barcelone de 1930 ", avec une référence sur la page de titre de l'édition de 1931 et de toutes les postérieures au prix obtenu également à l'exposition de Séville ${ }^{36}$. D'autre part, à la publicité (franche ou subreptice) dans les journaux et dans d'autres publications périodiques pour le grand public il a réussi à joindre quelques comptes rendus dans des revues spécialisées, telles que le Bulletin hispanique (no $35: 2,1933$, p. 205-206), où Georges Cirot, malgré quelques critiques ponctuelles, termine ses commentaires par «En somme, dictionnaire à recommander ». Du reste, certaines des observations critiques de Cirot ont été prises en compte par Reyes dans l'édition de 1934, ce qui lui a valu un nouveau compte rendu de Cirot dans Bulletin hispanique ( $\mathrm{n}^{\circ} 36: 3,1934$, p. 392), encore plus favorable que le précédent ${ }^{37}$. Toutefois, il s'est agi là de retouches mineures par rapport à l'ensemble des modifications auxquelles l'auteur a soumis son ouvrage au cours de ses premières rééditions. 


\subsection{La partie français-espagnol} position intermédiaire parmi les dictionnaires bilingues du même genre en ce qui concerne l'étendue de sa nomenclature. Comme l'auteur l'annonce sur la page de titre, l'ouvrage contenait dès le début un fort pourcentage de mots de spécialité. Il est donc loisible de se demander pourquoi il a décidé de l'élargir, au risque d'en élever le prix d'achat. Nous pensons que l'une des principales raisons en a été sa volonté de le présenter comme le plus riche des dictionnaires manuels français-espagnol de son temps. En fait, parmi ceux-ci, le véritable concurrent de celui de Reyes était à cet égard le dictionnaire de Toro y Gómez (1905), qui, commercialisé par la maison Armand Colin, se présentait sous un format très similaire ${ }^{39}$, mais dont la nomenclature était plus étendue que celle de Reyes du fait que cet auteur avait été le réviseur du dictionnaire encyclopédique bilingue de Salvá (1856), appartenant à la maison Garnier : rien que pour les entrées situées entre $L$ et Lazzo, le dictionnaire de Toro y Gómez ( $\left.{ }^{3} 1912\right)$ offre 151 lemmes que l'on ne trouve pas dans celui de Reyes $\left({ }^{1} 1926,{ }^{2} 1929,{ }^{3} 1930\right)$, alors que les lemmes présents dans les premières éditions du dictionnaire de Reyes mais absents dans celui de Toro y Gómez ne sont que 23, qui se réduisent à seulement 6 si l'on en exclut les participes passés et les noms propres, généralement non lemmatisés par Toro y Gómez. En ajoutant 109 lemmes nouveaux commençant par La- dans l'édition de 1934 - et une proportion similaire dans le reste de l'ouvrage -, Reyes obtient donc un dictionnaire parfaitement comparable quant au format et à la microstructure avec celui de Toro y Gómez (61923), mais le dépassant en nombre d'entrées ${ }^{40}$.

21 Or, curieusement, cet enrichissement du dictionnaire de Reyes a été surtout obtenu grâce à celui de Toro y Gómez ${ }^{41}$, sa source fondamentale pour établir sa nomenclature française de 1926 ainsi que pour l'augmentation à laquelle il l'a soumise en 1934. Notre auteur ne ment certes pas lorsqu'il déclare dans sa préface de 1926 qu'il a consulté d'autres dictionnaires pour rédiger le sien; c'est particulièrement évident en ce qui concerne celui de Darbas-Igon-Mareca (21896) et celui, à caractère encyclopédique, dû à Fernández Cuesta ( $\left.{ }^{1} 1885-1886-1887,{ }^{2} 1921\right)$, auxquels il a aussi puisé un certain nombre d'entrée nouvelles en 1934, cette fois-ci avec la contribution également du dictionnaire 
de Toro y Gisbert (1926). Mais il n'en reste pas moins vrai que leur influence demeure somme toute marginale si on la compare à celle de Toro y Gómez, son guide essentiel : le reste des dictionnaires lui ont surtout servi à différencier son ouvrage de sa source principale pour la partie français-espagnol.

\subsection{La partie espagnol-français}

Il en va autrement en ce qui concerne la partie espagnol-français. L'Académie espagnole a édité en 1927 la première édition de son dictionnaire manuel (Diccionario manual e ilustrado de la lengua española), dont une des principales caractéristiques - mis à part ses illustrations et sa réduction par rapport à son dictionnaire traditionnel, le Diccionario de la lengua castellana $\left({ }^{15} 1925\right)$ - a été l'incorporation d'un très grand nombre de vocables propres à l'espagnol d'Amérique. C'est justement l'évidente présence de ce genre de termes dans la partie espagnol-français du dictionnaire de Reyes qui en constitue l'un des traits saillants, ce qui dérive du simple fait qu'il en a établi la nomenclature en prenant comme source principale le Diccionario manual académique. Reyes l'avoue d'ailleurs ouvertement sur la page de titre et dans la préface de la seconde partie comme garantie de modernité et de fiabilité. Ayant caché les sources bilingues de sa première partie, il n'a à redouter, en revanche, aucune concurrence de la part du nouveau dictionnaire académique ; bien au contraire, le citer comme point de départ ne pouvait que lui rapporter des bénéfices aussi bien aux yeux de ses clients espagnols que, surtout, aux yeux de sa clientèle francophone, à laquelle il adressait particulièrement cette partie espagnol-français ${ }^{42}$.

Évidemment, le dictionnaire manuel de l'Académie espagnole pouvait procurer à notre auteur les lemmes pour établir sa nomenclature espagnole, mais il avait besoin de dictionnaires bilingues pour les correspondances françaises. Sans surprise, il a eu recours pour les trouver à sa source principale pour la première partie, le dictionnaire de Toro y Gómez, avec l'appoint, surtout, de l'ouvrage encyclopédique de Fernández Cuesta et du dictionnaire manuel de Darbas/Igon/Mareca. Au passage, il y a glané certains termes qui ne figuraient pas comme entrées dans le Diccionario manual de l'Académie espagnole ${ }^{43}$. Dès le début, par conséquent, la partie espagnol-français du dictionnaire de Reyes a été bien plus riche en nombre d'entrées que la partie correspondante des dictionnaires similaires au sien, ce qui pourrait expliquer qu'il ait atteint sa forme pratiquement définitive dès l'édition de $1930^{44}$, alors qu'il faut attendre celle de 1934 pour ce qui est de la partie inverse.

\section{Conclusion}

Rafael Reyes est l'un des meilleurs représentants des professeurs de l'enseignement secondaire qui, depuis le XIX siècle, ont ajouté à la publication de manuels pour l'apprentissage du français l'édition d'un dictionnaire bilingue. Le sien, grâce à son long parcours éditorial depuis 1926, a été le plus populaire en Espagne pendant un demisiècle. C'est une des raisons principales pour lesquelles le lycée de la ville natale de Reyes porte son nom depuis 1977. En outre, les autorités municipales de cette même ville ont décidé le 20 janvier 2006 de lui octroyer la distinction de citoyen d'honneur, après avoir collaboré financièrement avec les autorités provinciales à une réédition (2003) en fac-similée ${ }^{45} d u$ dernier tirage de son dictionnaire $\left({ }^{41} 1979\right)$. À notre 
connaissance, aucun autre dictionnaire franco-espagnol contemporain n'a joui à ce jour de cette même prérogative.

\section{BIBLIOGRAPHIE}

BRUÑA CUEVAS, Manuel (2005). « Histoire des transcriptions phonétiques dans les dictionnaires français-espagnol et espagnol-français ». Cahiers de Lexicologie, 87/2, 97-140.

CASTEllano, Philippe (2015). « Francia, España, Hispanoamérica. Estrategias editoriales ante el mercado internacional del libro (1900-1914)». Cahiers de civilisation espagnole contemporaine, 2. En ligne : <http://ccec.revues.org/5546> (14 janvier 2016).

ESPINOSA SANSANO, María Dolores (2012). « De l'Abrégé de la Grammaire Françoise, de M. de Wailly, au Curso práctico de Francés, de Rafael Reyes. Une évolution? ». In Esperanza Bermejo Larrea, J. Fidel Corcuera Manso, Julián Muela Ezquerra (éd.). Communication et écritures. Autour de la linguistique et de la littérature françaises. Saragosse : Prensas de la Universidad de Zaragoza, 101-108.

FERNÁNDEZ FRAILE, María Eugenia \& SUSO LÓPEZ, Javier (1999). La enseñanza del francés en España (1767-1936). Estudio histórico : objetivos, contenidos, procedimientos. Grenade : Método.

GONZÁLEZ GARCíA, Juana María (2014). « Reyes Rodríguez, Rafael ». In JAE Educa, Diccionario de profesores de instituto vinculados a la JAE (1907-1936). En ligne : <http://ceies.cchs.csic.es/? q=content/reyes-rodr\%C3\%ADguez-rafael> (7 janvier 2016).

JUNTA PARA AMPLIACIÓN DE ESTUDIOS E INVESTIGACIONES CIENTÍFICAS (1935). Memoria correspondiente a los cursos 1933 y 1934. Madrid : Góngora.

LÓPEZ BARRERA, Joaquín (1908). Apuntes para una historia de la enseñanza de la lengua francesa en España. Cuenca : C. León.

ROSA REINERO, José Luis de la (2003). «Datos biográficos de un ilustre cartayero : D. Rafael Reyes Rodríguez ». In Rafael Reyes. Diccionario francés-español y español-francés, reproduction en facsimilé de l'édition de 1979. [Huelva] : Diputación de Huelva, Ayuntamiento de Cartaya, XIX-XXI.

\section{NOTES}

1. D'après Rosa Reinero (2003 : XIX), Reyes est mort à Alicante le 28 février 1944, ce qui nous semble peu probable.

2. Gaceta de Madrid du 2 août 1906.

3. Il a dispensé des cours d'anglais jusqu'au 7 novembre 1904 au Centro Gallego de Madrid (voir le journal El Imparcial, 6/10/1904, p. 4, et la Gaceta de instrucción pública, 7/11/1904, p. 4) et des cours de français à l'Ateneo de la même ville (La Correspondencia de España, 6/1/1906, p. 3 ; ABC, 18/2/1906, p. 6).

4. Il a été, par exemple, rédacteur habituel de la Gaceta de instrucción pública et a publié l'article «Un paseo por Bretaña » (une promenade en Bretagne) dans la revue Por esos mundos (1/11/1905, p. 12-19). 
5. Il a obtenu ce poste après des échecs à quelques autres concours nationaux de l'enseignement secondaire, auxquels il s'est présenté au moins depuis 1902.

6. Sur cette grammaire, voir Fernández Fraile \& Suso López (1999 : 419).

7. Son père, Isidoro Reyes, mourra à Cartaya le 7 juillet 1908 ( $A B C, 26 / 71908$; Gaceta de instrucción pública y bellas artes, 30/7/1908, p. 8). Auparavant, Reyes, quoique établi à Madrid, revenait périodiquement dans sa ville natale; de fait, pendant l'été 1904 il a prononcé la conférence « La enseñanza obrera » (l'enseignement ouvrier) au Cercle républicain de cette localité.

8. Ses deux ouvrages sur la matière déjà publiés connaîtront des rééditions à l'époque : en 1907, sa grammaire; en 1910, son anthologie d'extraits littéraires. Tous deux obtiendront le 17 juin 1910 un avis favorable de l'Académie espagnole (Gaceta de instrucción pública y bellas artes, 15/11/1910, p. 2).

9. Il s'agit d'un organisme créé par l'État en 1907 dans le cadre de l'Institución Libre de Enseñanza. Son but était de promouvoir, au moyen de bourses, les stages à l'étranger des professeurs et des chercheurs espagnols.

10. Le titre qu'il assignait à son travail était Relaciones diplomáticas de España con las demás potencias durante la Regencia de María Cristina (Archives de la Junta, JAE 122/127).

11. D’après González García, les Archives générales de l'Administration espagnole - AGA, 5 (17) 32/08462 - 5873/1 - conservent un autre travail inédit de Reyes ; il est daté de Madrid le 6 avril 1909 et s'intitule (nous traduisons de l'espagnol) Racine et la Grammaire ou Observations grammaticales sur le lexique employé par Racine dans ses œuvres.

12. Il se présente sur la page de titre de la plupart de ses publications comme avocat et comme membre de l'Académie espagnole de Jurisprudence.

13. Gaceta de Madrid, 20/4/1919. Son premier congé, d'un mois, lui a été accordé en 1917 ( $L a$ Mañana, 23/10/1917, p. 4).

14. Un bref travail de ce professeur (1908, 19 pages) constitue la première publication consacrée exclusivement à l'histoire de l'enseignement du français en Espagne.

15. Reyes a fait appel plusieurs fois contre les décisions ministérielles. La Gaceta de Madrid (équivalent du Journal Officiel), par exemple, a publié le 23 février 1928 un arrêté ministériel par lequel Reyes obtenait gain de cause dans sa réclamation pour remplacer le titulaire nommé pour le jury d'un concours de postes de professeurs qui avait renoncé à sa charge.

16. Nous indiquerons le numéro d'édition de chaque ouvrage au moyen d'un chiffre en exposant précédant sa date de parution. S'agissant, par exemple, du Traductor de Reyes, “ ${ }^{2} 1923$ » se réfère à sa deuxième édition, parue en 1923.

17. Il s'agit, pour le premier niveau, de la réédition de ${ }^{7} 1926$ et, pour le second, des rééditions de ${ }^{4} 1923,{ }^{5} 1924$ et ${ }^{6} 1926$.

18. En 1929, il avait aussi un logement à Séville (5, rue Cuesta del Rosario), mais, en 1932, il s'hébergeait à l'hôtel Royal de cette ville et situait son domicile à Madrid, 63, rue Vicente Blasco Ibáñez. Peut-être du fait que cette dernière rue a porté le nom de "Princesa » avant et après la Deuxième République espagnole, beaucoup de répertoires bibliographiques et de catalogues bibliothécaires donnent «Blasco Ibáñez » comme le nom de la maison d'édition des ouvrages de Reyes, alors qu'il y consignait ce nom tout simplement comme l'adresse à laquelle il fallait en faire les commandes.

19. Archives de la Junta (JAE 122/127). Étant donné son salaire comme professeur, il ne demande pas une bourse pour faire son stage; il demande tout simplement, comme le prévoyait la normative en vigueur pour les plus fortunés, d'avoir le même statut de chercheur à l'étranger qu'avaient les véritables boursiers. Voir Junta para Ampliación de Estudios (1935 : 189).

20. Comme nous le verrons, ce n'est pas, malgré quelques retouches, la partie espagnol-français de son dictionnaire qu'il remaniera vraiment à l'époque, mais la partie français-espagnol. Par ailleurs, Reyes a prononcé deux conférences pendant son séjour en France, toutes deux sur le théâtre espagnol contemporain: la première, le 21 janvier 1933 à l'Athénée de Bordeaux, à 
l'invitation de l'Amicale démocratique franco-espagnole (La Vanguardia, 22/1/1933, p. 29 ; El Sol, 22/1/1933, p. 7) ; l'autre, le $1^{\text {er }}$ février 1933 à la Sorbonne, à l'invitation de la Société pour la promotion des langues étrangères en France (La Vanguardia, 2/2/1933, p. 22; La Libertad, 2/2/1933, p. 5).

21. Archives de la Junta (JAE 122/127).

22. Datant de 1906 , cet ouvrage connaît alors ses $10^{\mathrm{e}}(1930), 11^{\mathrm{e}}(1931), 12^{\mathrm{e}}$ (1932) et $13^{\mathrm{e}}(1936)$ éditions en ce qui concerne son premier niveau, ainsi que ses $9^{\mathrm{e}}(1931), 10^{\mathrm{e}}(1932), 11^{\mathrm{e}}(1933), 12^{\mathrm{e}}$ (1934) et $13^{\mathrm{e}}$ (1936) éditions pour le second.

23. Avec une première édition en 1906, les Trozos en sont à leur $8^{\mathrm{e}}$ édition en 1930, à laquelle vient s'ajouter, avec certaines modifications dans le titre et le contenu, la $9^{\mathrm{e}}$ en 1935.

24. ${ }^{1} 1926,{ }^{2} 1928,{ }^{3} 1929,,{ }^{4} 1930,{ }^{5} 1931,{ }^{6} 1932,{ }^{7} 1933,{ }^{8} 1934,{ }^{9} 1935,{ }^{10} 1936$.

25. C'est cette importance du dictionnaire du point de vue économique qui pourrait expliquer les attaques continues qu'il formule dans les préfaces de ses ouvrages contre les auteurs qui incluaient un vocabulaire bilingue fondamental dans leurs anthologies de morceaux choisis pour le secondaire. Lui-même, pourtant, en avait inclus un dans les premières éditions de son Traductor (p. 239-261 en ${ }^{2} 1923$, par exemple) ; il l'a supprimé dès la parution de son dictionnaire, le remplaçant par une note, réitérée de nombreuses fois, où il conseille d'avoir recours à ce dernier pour surmonter les difficultés de compréhension posées par les extraits.

26. Ce n'était pas la première fois qu'il adaptait ses ouvrages aux différents plans d'études qui se succédaient. Comme nous le verrons, ce ne sera pas non plus la dernière.

27. La literatura francesa ilustrada con textos. Tome I : Desde los orígenes hasta fines del siglo XVII (Des origines à la fin du XVII ${ }^{\mathrm{e}}$ siècle, 1935). Tome II : Desde el siglo XVIII hasta nuestros días (Du XVIII siècle à nos jours, 1936).

28. Reyes a dédié sa grammaire anglaise (1910) au libéral José Canalejas, premier ministre espagnol à l'époque, dont il dit partager les idées; or celui-ci a réprimé toutes les révoltes républicaines qui se sont produites sous son mandat (voir néanmoins notre note 7). De plus, notre auteur semble ne pas avoir eu d'activité politique pendant la période républicaine. Par ailleurs, les définitions de langue et de dialecte dans le premier cours de sa grammaire - qui était en 1932 le livre de texte de français au lycée de Barcelone - avaient été publiquement condamnées par la Fédération nationale des étudiants de Catalogne (La Vanguardia, 16/12/1932, p. 10) : Reyes y rabaissait le catalan à la catégorie de dialecte. Or, ce genre de définitions ne pouvait que plaire aux nouvelles autorités franquistes.

29. Boletín Oficial del Estado du 17 octobre 1937.

30. Reyes annonce dans l'édition de 1938 de son dictionnaire qu'il prépare la publication de La Enseñanza en España. À notre connaissance, cet ouvrage, dont il avait déjà forgé le projet dès 1930 (préliminaires de la $8^{\mathrm{e}}$ réédition de ses Trozos), n'a jamais été mis sous presse. Il ne s'agit pas là d'un cas unique; un Cours pratique d'espagnol et un Curso práctico de inglés, annoncés dans les préliminaires de l'édition de 1923 de son Traductor, ne verront pas non plus le jour (même son Curso práctico de francés n'a connu une deuxième édition qu'en 1933, dix ans après la première, contrairement à ce qui était habituel pour le reste de ses ouvrages).

31. Boletín Oficial del Estado du 14 août 1940.

32. Boletín Oficial del Estado du 25 février 1941.

33. Seul le dernier tirage (1979) ne sera plus publié par la maison d'édition fondée par l'auteur dans les années 1940 (Editorial Reyes), mais par la librairie et maison d'édition Hernando.

34. L'édition de 1928 se présente avec une première partie français-espagnol, imprimée par Sáez Hermanos, et une seconde espagnol-français, imprimée par Huelves y Cía.

35. Reyes visait les élèves du secondaire, même si, dans sa brève préface de 1926, il dit adresser son ouvrage à tout Espagnol devant lire, traduire ou écrire le français : « [...] para todo el que tenga que leer o escribir el francés [...]», pour « todo español que tenga que traducir o escribir el francés ". 
36. «Obra premiada con Medalla de Oro en la Exposición Internacional de Barcelona y en la Iberoamericana de Sevilla de 1930. »

37. Quoique vivement critiquée par Cirot, Reyes n'a pas éliminé de son dictionnaire la prononciation figurée dont étaient assortis tous les lemmes français. Sur le système de transcriptions de notre auteur, voir Bruña Cuevas (2005).

38. Les lemmes LAIZE, LAMIER, LAPS et LARMOIEMENT deviennent respectivement LAIZE 0 LAISE, LAMIER 0 LAMIER BLANC, LAPS $O$ LAPSE, LARMOIEMENT $o$ LARMOÎMENT ; les lemmes LARVÉE, LARINGITE et LARINGOLOGIE sont corrigés en LARVÉ, LARYNGITE, LARYNGOLOGIE; le lemme LACOSTE apparaît en 1934 à sa place alphabétique attendue, ce qui n'était pas le cas dans les premières éditions.

39. Le dictionnaire de Darbas et Igon, révisé par Mareca ( $\left.{ }^{2} 1896\right)$, était également similaire à ceux de Toro y Gómez et de Reyes, mais il a connu sa dernière édition française vers 1914.

40. Quelques chiffres supplémentaires peuvent être illustratifs à cet égard. Toujours en prenant comme échantillon les lemmes français compris entre l'entrée $L$ et la dernière entrée commençant par La-, le dictionnaire de Gildo incluait, dans son édition de 1906, 293 lemmes ; ils sont 184 dans celui de Tolhausen ( $\left.{ }^{7} 1914\right), 277$ dans celui d'Alcalá-Zamora et Antignac (édition de 1926) et 333 dans celui de Toro y Gisbert (édition de 1926). Les deux dictionnaires manuels les plus riches en nombre d'entrées françaises, ceux de Darbas/Igon/Mareca (édition de 1906) et de Toro y Gómez (rééditions des années 1910), en présentaient respectivement 380 et 381 . Comme nous l'avons dit, le dictionnaire de Reyes, pour le même échantillon, a été augmenté de 355 lemmes en ${ }^{1} 1926$ à 465 en 1934.

41. Bien que son dictionnaire ait continué à être publié par la maison Armand Colin, Toro y Gómez avait changé l'orientation de ses activités dès 1912, date à laquelle il s'était établi à Buenos Aires, où il est mort en 1922. Reyes n'avait donc pas à craindre la réaction critique de ce lexicographe. Sur lui et sur son œuvre, voir Castellano (2015).

42. En commentant dans la préface de l'édition de 1938 la présence dans l'ouvrage d'un appendice sur le régime prépositionnel des verbes espagnols, il déclare que, par-là, son ouvrage "peut être considéré, mieux que jamais, comme un instrument de travail indispensable pour tous les hispanisants et pour tous les élèves d'Espagnol de la France, de la Belgique et de la Suisse française » qui lui font l'honneur de le consulter.

43. Entre le lemme $L$ et le dernier lemme commençant par La- (LAZULITA) de la partie espagnolfrançais de Reyes, on compte 518 entrées, toutes, sauf 19, présentes dans le dictionnaire manuel académique. Or, si l'on exclut de ces 19 entrées les noms propres (souvent présents chez Darbas/ Igon/Mareca) et certaines variantes morphologiques correspondant à des lemmes donnés par le dictionnaire académique, l'apport réel de Reyes en comparaison avec ce dernier se réduit à dix lemmes (LABIODENTAL, LABRO, LADRONCILLO, LAICISACIÓN, LAICISAR, LANCEAR, LARINGOLOGÍA, LASQUENETE, LÁUREA ET LAUREADO), six d'entre eux provenant soit de Toro y Gómez (LABIODENTAL, LADRONCILLO, LANCEAR et LÁUREA) soit de Fernández Cuesta (LABRo et LAUREADO).

44. Dans notre échantillon, les entrées comprises entre $L$ et LAZULITA sont exactement les mêmes dans la dernière édition de 1979 et dans la première de 1928. Les seules variations concernant la nomenclature espagnole entre 1928 et les rééditions des années 1930 (et des décennies postérieures) ont trait à la correction de l'accentuation: les lemmes LAMPREílLA, LANío, LARINGOSCOPíA et LÁUREO, sans accent en 1928, le porteront par la suite, conformément à leur orthographe dans le Diccionario manual de l'Académie espagnole.

45. Avec une préface d’E. Suárez Sánchez et C. Ramírez Gómez et quelques renseignements biographiques dus à J. L. de la Rosa Reinero. 


\section{RÉSUMÉS}

Rafael Reyes (1879-1947) a publié un certain nombre de grammaires et d'anthologies pour l'enseignement de la langue française en Espagne. Mais l'ouvrage qui l'a rendu vraiment célèbre est son dictionnaire français-espagnol et espagnol-français (1926-1928). Dans ce travail, nous traçons d'abord la vie de l'auteur telle qu'elle nous est révélée par des documents d'archives et des articles de journaux; nous énumérons les postes de travail qu'il a occupés et les circonstances qui rendent compte des nombreuses rééditions de ses livres. Puis nous centrons notre attention sur son dictionnaire bilingue, le plus populaire dans son genre en Espagne depuis les années 1930 jusqu'aux années 1970. Nous analysons les causes de sa vitalité éditoriale et de ses remaniements et révélons les sources où l'auteur a puisé.

Rafael Reyes (1879-1947) published a certain number of grammars and anthologies for teaching the French language in Spain. But the book which made him really famous was his FrenchSpanish and Spanish-French dictionary (1926-1928). In this work, we first trace the life of Reyes such as it is revealed by archival documents and newspaper articles ; we list the jobs he held and the circumstances which explain the many editions of his books. Then we centre our attention on his bilingual dictionary, the most popular of its kind in Spain since the 1930s until the 1970s. We analyse the causes of its editorial success and of its changes and reveal the sources of the author.

\section{INDEX}

Mots-clés : Rafael Reyes, enseignants du secondaire, dictionnaires français-espagnol, manuels espagnols de FLE, français pour Espagnols

Keywords : Rafael Reyes, teachers of secondary education, French-Spanish dictionaries, French teaching books, French for Spanish speakers

\section{AUTEUR}

\section{MANUEL BRUÑA CUEVAS}

Université de Séville mbruna@us.es 